\title{
Bipartite entanglement of multipartite coherent states using quantum network of beam splitters
}

\author{
M. Daoud ${ }^{a, \text { }^{*}}$ and E. B. Choubabi $\oplus$ \\ ${ }^{a}$ Max Planck Institute for Physics of Complex Systems, Nöthnitzer Str. 38, \\ D-01187 Dresden, Germany \\ ${ }^{b}$ Department of Physics, Faculty of Sciences, Ibn Zohr University, \\ PO Box 8106, 80006 Agadir, Morocco \\ ${ }^{c}$ Theoretical Physics Group, Faculty of Sciences, Chouaib Doukkali University, \\ PO Box 20, 24000 El Jadida, Morocco
}

\begin{abstract}
We investigate the generation of multipartite entangled $S U(k+1)$ coherent states using a quantum network involving a sequence of $k$ beam splitters. We particularly investigate the entanglement in multipartite $S U(2)$ coherent states $(k=1)$. We employ the concurrence as measure of the degree of bipartite entanglement.
\end{abstract}

*daoud@pks.mpg.de, m_daoud@hotmail.com

†choubabi@gmail.com 


\section{Introduction}

Due to seminal works on quantum teleportation [1], superdense coding [2] and quantum key distribution [3], entanglement in multipartite quantum systems began to be recognized as valuable resource for performing communication and computational tasks [4, 5, 6]. This motivated the considerable interest in the development of a quantitative theory of entanglement and the definition of its basic measure (concurrence, entanglement of formation and linear entropy [7, , 9, 10]). The physical implementation of entangled states is then of major importance and several different physical systems have been considered. We mention the generation of entangled electromagnetic states using type I or type II parametric down conversion [11. Another experimentally accessible device which can be used to generate optical entangled states is the beam splitter [12, 13, 14, 15]. In quantum optics the action of a beam splitter, which is essentially a mixer of two electromagnetic modes, can be represented by a unitary operator relating the input and the output states. In general, the output state is a superposition of the Fock states which is entangled, except the harmonic oscillator coherent states who do not exhibit entanglement when passed through one arm of 50:50 beam splitter while the second arm is left in the empty vacuum state [16]. The beam splitter device provides a very useful technique to investigate the entanglement of nonorthogonal states as for instance the entangled coherent states introduced in [13, 14, 17, 18, 19]. They found a notable success in the context of quantum teleportation [20, 21], quantum information processing [22, 23], tests of local realism [13] and very recently an approach for quantum repeaters with entangled coherent states was proposed in [24]. All those applications explain their intensive investigation. In this sense, the entanglement behavior of $S U(2)$ spin coherent states, when passed through a beam splitter, has been previously considered in [25]. Similar study was done in [26] for $S U(1,1)$ coherent states defined in an infinite dimensional Hilbert space. Entangled quantum systems can exhibit correlations that cannot be explained on the basis of classical laws and the lack of entanglement in a collection of states is clearly a signature of classicality [25]. The fact that the coherent states minimize the quantum fluctuations [27, 28] and subsequently present semi-classical behavior, constitutes the main motivation to use them for the understanding of bipartite [13, 14, 17, 18, 19, 29, 30] as well as the multipartite entanglement of harmonic oscillator coherent states [31, 32].

In this paper, we shall be interested in the bipartite entanglement of generalized coherent states generated from the quantum electromagnetic states passing through a quantum network of $k$ beam splitters. We show that under some special assumptions, one can generate $S U(2)$ for $k=2, S U(3)$ for $k=3$ or more generally $S U(k+1)$ multipartite coherent states which are labeled by continuous variables related to reflection-transmission coefficients of the beam splitters.

The outline of the paper is as follows. In section 2, we define the action of a quantum network of $k$ beam splitters. We show that this action leads $S U(k+1)$ coherent states, in the Perelomov sense, labeled by complex variables related to reflection and transmission parameters of beam splitters. This means that multi-port beam splitters are applicable in optical realizations of coherent states associated with higher symmetries. In section 3, we consider the superpositions of equally weighted $S U(2)$ multipartite coherent states arising from two quantum networks of beam splitters characterized by different reflection-transmission parameters. By equally weighted, we mean that each multipartite wave function is equally balanced with the other element in the superposition. We divide the entire 
system into two subsystems to discuss the bipartite entanglement. We employ the concurrence 33 ] to determine the degree of entanglement. We consider the pure and mixed state cases. Concluding remarks close this paper.

\section{Quantum network of beam splitters}

As mentioned above, the study of entangled states in the last decade has revived interest in the beam splitter because this device offers us an simple way to probe the quantum nature of electromagnetic field by means of simple experiments. We recall that the beam splitter is an optical element with two input ports and two output ports which, in some sense, governs the interaction of two harmonic oscillators. The input and output boson operators are related by a unitary transformation which may be viewed as an element of the $S U(2)$ group. Recently, a quantum network of beams splitters was used to create multi-particle entangled states of continuous variables [34] and also multi-particle entangled coherent states [32] associated with the usual harmonic oscillator algebra.

\section{$2.1 \quad$ Fock states output}

We shall First discuss a simplest beam splitters combination that enables the generation of coherent states associated with the Lie algebra $s u(k+1)$ using $k+1$ different electromagnetic field modes $(k \in \mathbb{N}-\{0\})$. To this end, we define a sequence of $k$ beam splitters (" $k$-beam splitters") acting as follows. The output of the first beam splitter, which is a superposition of the modes 0 and 1 , is injected into the first arm of the second beam splitter while the second arm receives modes of quantum electromagnetic field labeled as 2. The output state of the second beam splitter is then injected in the the first port of the third beam spitter while the other port receives the photons in the mode 3. This scheme can be generalized to an arbitrary number of beam splitters such that the output state of the each beam splitter, of the sequence under consideration, is inserted in one port of the next beam splitter while another electromagnetic mode is inserted into the other input port. The figure 1 give the schematics of this combination of beam splitters.

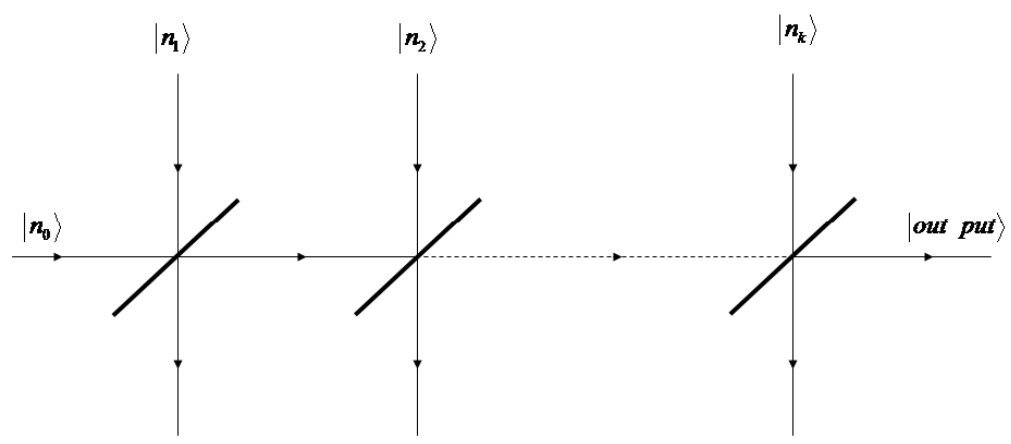

Fig. 1: The sequence of $k$ beam splitters.

Then the unitary transformation to realize this can be expressed as the product of a sequence of beam splitter tansformations. It is given by

$$
\mathcal{U}_{k}=\mathcal{B}_{k-1, k}\left(\theta_{k}\right) \mathcal{B}_{k-2, k-1}\left(\theta_{k-1}\right) \cdots \mathcal{B}_{0,1}\left(\theta_{1}\right)
$$


where the operators

$$
\mathcal{B}_{l, l+1}\left(\theta_{l+1}\right)=\exp \left(\frac{i}{2} \theta_{l+1}\left(a_{l}^{+} a_{l+1}^{-}+a_{l}^{-} a_{l+1}^{+}\right)\right)
$$

are the ordinary $S U(2)$ beam splitters for $(l=0,1,2, \cdots, k-1)$ and the angles $\theta_{l}$ are related to reflection and transmission coefficients as follows

$$
t_{l}=\cos \frac{\theta_{l}}{2} \quad r_{l}=\sin \frac{\theta_{l}}{2} .
$$

In the equation (2),$a_{l}^{+}$and $a_{l}^{-}$stand the creation and annihilation operators of $k+1$ independent bosonic oscillator. They satisfy the usual commutation rules

$$
\left[a_{l}^{-}, a_{l^{\prime}}^{+}\right]=\delta_{l l^{\prime}}, \quad\left[a_{l}^{-}, a_{l^{\prime}}^{-}\right]=\left[a_{l}^{+}, a_{l^{\prime}}^{+}\right]=0, \quad l, l^{\prime}=0,1,2, \cdots k .
$$

As Hilbert space let chose the infinite dimensional Fock space generated by multi-mode states

$$
\mathcal{H}=\mathcal{H}_{0} \otimes \mathcal{H}_{1} \cdots \otimes \mathcal{H}_{k}=\left\{\left|n_{0}, n_{1}, \cdots, n_{k}\right\rangle, n_{i} \in \mathbb{N}\right\}
$$

If the input state is given by $\left|n_{0}, n_{1}, \cdots, n_{k}\right\rangle$, the action of the operator $\mathcal{U}_{k}$ is given by the following Fock states superposition

$$
\mathcal{U}_{k}\left|n_{0}, n_{1}, \cdots, n_{k}\right\rangle=\sum_{m_{0}, m_{1}, \cdots, m_{k}} C_{n_{0}, n_{1}, \cdots, n_{k}}^{m_{0}, m_{1}, \cdots, m_{k}}\left|m_{0}, m_{1}, \cdots, m_{k}\right\rangle
$$

where the coefficients $C$ stand for the matrix elements of the unitary operator $\mathcal{U}_{k}$. In general the output is a $(k+1)$-particle entangled state. On the other hand, the action of the unitary operator $\mathcal{U}_{k}$ on the state $\left|n_{0}=n, 0, \cdots, 0\right\rangle$ gives

$\mathcal{U}_{k}|n, 0, \cdots, 0\rangle=\mathcal{C} \sum_{n_{1}=0}^{n} \sum_{n_{2}=0}^{n_{1}} \cdots \sum_{n_{k}=0}^{n_{k-1}} \frac{\xi_{1}^{n_{1}} \xi_{2}^{n_{2}} \cdots \xi_{k}^{n_{k}} \sqrt{n !}}{\sqrt{\left(n-n_{1}\right) !\left(n_{1}-n_{2}\right) ! \cdots\left(n_{k-1}-n_{k}\right) ! n_{k} !}}\left|n-n_{1}, n_{1}-n_{2}, \cdots, n_{k}\right\rangle$

where the normalization constant is given by

$$
\mathcal{C}=\left(1+\left|\xi_{1}\right|^{2}+\left|\xi_{1}\right|^{2}\left|\xi_{2}\right|^{2} \cdots+\left|\xi_{1}\right|^{2}\left|\xi_{2}\right|^{2} \cdots\left|\xi_{k}\right|^{2}\right)^{-\frac{n}{2}}
$$

and the new variables $\xi$ are defined by

$$
\xi_{l}=i t_{l+1} \frac{r_{l}}{t_{l}} \quad \text { for } \quad l=1,2, \cdots, k-1 \quad \text { and } \quad \xi_{k}=i \frac{r_{k}}{t_{k}}
$$

in terms of the reflection and transmission coefficients of the beam splitters constituting the network. Then, the output state (7) turns out to be the $S U(k+1)$ coherent state associated with the completely symmetric representation labeled by the integer $n$. Indeed, the bilinear product of creation and annihilation modes realizes, à la Schwinger, the generators of the Lie algebra $s u(k+1)$ and the operator $\mathcal{U}_{k}$ is nothing but a unitary displacement operator which acting on the highest weight vector $\left|n_{0}=n, 0, \cdots, 0\right\rangle$, gives the $S U(k+1)$ coherent states in the Perelomov sense (see for instance [35] for more details). This is clarified in the what follows. 


\section{2 $S U(k+1)$ coherent states}

In this subsection we discuss the relationship between the states (17) and the $S U(k+1)$ coherent states. To do this, it is interesting to note that the states (7) write as an expansion of the number states belonging the the restricted Fock space

$$
\mathcal{F}=\left\{\left|n_{0}, n_{1}, n_{2} \cdots, n_{k}\right\rangle, n_{0}+n_{1}+n_{2}+\cdots+n_{k}=n\right\}
$$

which is finite dimensional. In order to show that (7) are $S U(k+1)$ coherent states, we realize the $s u(k+1)$ generators as bilinear products in creation and annihilation operators. They are given by

$$
e_{i}^{-}=a_{i}^{+} a_{i+1}^{-} \quad e_{i}^{+}=a_{i}^{-} a_{i+1}^{+} \quad i=0,1, \cdots, k,
$$

and the Cartan generators

$$
h_{i}=a_{i}^{+} a_{i}^{-}-a_{i+1}^{+} a_{i+1}^{-}, \quad i=0,1, \cdots, k-1 .
$$

The generators $e_{i}^{-}$and $e_{i}^{+}$are the so-called Weyl operators. The generators of $s u(k+1)$ with a non trivial action (non vanishing and non diagonal) on the fiducial vector (or the highest weight vector of the symmetric representation) are

$$
t_{i}^{+}=a_{0}^{-} a_{i}^{+} \quad t_{i}^{-}=a_{0}^{+} a_{i}^{-} \quad i=1,2, \cdots, k .
$$

They can be defined from the $s u(k+1)$ generators as follows

$$
\begin{array}{ll}
t_{1}^{+}=e_{0}^{+}, & t_{i+1}^{+}=\left[e_{i}^{+}, t_{i}^{+}\right], \\
t_{1}^{-}=e_{0}^{-}, & t_{i+1}^{-}=\left[t_{i}^{-}, e_{i}^{-}\right],
\end{array}
$$

for $i=1,2, \cdots, k-1$. Using the raising and lowering operators $t_{i}^{+}$and $t_{i}^{-}$, the states (77) can be shown to have the displacement forms. Namely, they are created by the action of the unitary displacement operators in $S U(k+1)$ acting on the state $\left|n=n_{0}, 0, \cdots, 0\right\rangle$ (the vacuum). Indeed, it is simply verified that the action of the unitary operator

$$
D\left(\xi_{1}, \xi_{2}, \cdots, \xi_{k}\right)=\exp \left(\sum_{i=1}^{k}\left(\xi_{i} t_{i}^{+}-\bar{\xi}_{i} t_{i}^{-}\right)\right)
$$

on the state $\left|n=n_{0}, 0, \cdots, 0\right\rangle$ gives the states (17). This is exactly the Perelomov definition of coherent states for Lie algebras. Thus, it is clear that the output states (17) constitutes a special class of $S U(k+1)$ coherent states.

To close this subsection, it is important to stress that the generation of $S U(k+1)$ coherent states using the optical chain of $k$ beam splitters described above requires input radiation state with fixed number of photons. The experimental production of such interesting and highly non classical states has been investigated during the last decade (see [36] and references therein). Recently an important experimental advance was reported by Hofheinz et al in [37]. They gave the first experimental demonstration for generating photon number Fock states containing up to $n=6$ photons in a superconducting quantum circuit. 


\subsection{Contraction of $s u(k+1)$ algebra and Glauber coherent states}

At this stage, it is natural to ask about the relation between $S U(k+1)$ coherent states obtained above and the standard Glauber coherent for multi-mode electromagnetic fields. In this subsection, we shall show that he usual Glauber coherent states can be obtained from the $S U(k+1)$ as a special limiting case. To show this, we rewrite the coherent states (7) as

$$
\left|\zeta_{1}, \zeta_{2}, \cdots, \zeta_{k}\right\rangle=\mathcal{C} \sum_{n_{1}=0}^{n} \sum_{n_{2}=0}^{n-n_{1}} \cdots \sum_{n_{k}=0}^{n-\sum_{i=1}^{k} n_{i}} \frac{\zeta_{1}^{n_{1}} \zeta_{2}^{n_{2}} \cdots \zeta_{k}^{n_{k}} \sqrt{n !}}{\sqrt{\left(n-\sum_{i=1}^{k} n_{i}\right) ! n_{1} ! n_{2} ! \cdots n_{k} !}}\left|n-\sum_{i=1}^{k} n_{i}, n_{1}, n_{2}, \cdots, n_{k}\right\rangle
$$

where the new variables are defined by

$$
\zeta_{i}=\xi_{1} \xi_{2} \cdots \xi_{i} \quad i=1,2, \cdots, k
$$

Let $n \rightarrow \infty,\left|\zeta_{i}\right| \rightarrow 0$ in such a way that the product $n\left|\zeta_{i}\right|^{2}=\left|z_{i}\right|^{2}$ is fixed. In this limit, the coherent state (10) tends to

$$
\left|\zeta_{1}, \zeta_{2}, \cdots, \zeta_{k}\right\rangle \rightarrow|n\rangle \otimes\left|z_{1}\right\rangle \otimes\left|z_{2}\right\rangle \cdots \otimes\left|z_{k}\right\rangle
$$

where

$$
\left|z_{i}\right\rangle=\exp \left(-\left|z_{i}\right|^{2} / 2\right) \sum_{n_{i}=0}^{\infty} \frac{z_{i}^{n_{i}}}{\sqrt{n_{i} !}}\left|n_{i}\right\rangle
$$

are the usual Glauber coherent states. This limit can understood as contraction of $s u(k+1)$ algebra into $k$ independent Weyl-Heisenberg algebras. Indeed, using

$$
\begin{gathered}
t_{i}^{+}\left|n_{0}, n_{1}, \cdots n_{i}, \cdots, n_{k}\right\rangle=\sqrt{n_{0}\left(n_{i}+1\right)}\left|n_{0}, n_{1}, \cdots n_{i}+1, \cdots, n_{k}\right\rangle \\
t_{i}^{-}\left|n_{0}, n_{1}, \cdots n_{i}, \cdots, n_{k}\right\rangle=\sqrt{\left(n_{0}+1\right) n_{i}}\left|n_{0}+1, n_{1}, \cdots n_{i}-1, \cdots, n_{k}\right\rangle
\end{gathered}
$$

and the relation $n_{0}=n-\left(n_{1}+n_{2}+\cdots+n_{k}\right)$ satisfied by the states of the $S U(k+1)$ invariant Fock space $\mathcal{F}$, one can simply verify that for $n$ large

$$
t_{i}^{+} \sim \sqrt{n} a_{i}^{+} \quad t_{i}^{-} \sim \sqrt{n} a_{i}^{-}
$$

It follows that in this limiting case, we end up with $k$ commuting copies of usual harmonic oscillator and the states (10) gives

$$
\left|\zeta_{1}, \zeta_{2}, \cdots, \zeta_{k}\right\rangle \rightarrow \exp \left(\sum_{i=1}^{k}\left(z_{i} a_{i}^{+}-\bar{z}_{i} a_{i}^{-}\right)\right)|n, 0,0, \cdots, 0\rangle
$$

where the right hand coincides with $|n\rangle \otimes\left|z_{1}\right\rangle \otimes\left|z_{2}\right\rangle \cdots \otimes\left|z_{k}\right\rangle$ reflecting that, in this special case, the output state is completely separable.

\subsection{Generation of multipartite nonorthogonal states}

Here we provide an optical scheme to generate multipartite $S U(k+1)$ coherent states. It clear that with $p$ decoupled network of quantum beam splitters, on can generate a tensorial product of $S U(k+1)$ coherent states. For instance, the special case $k=1$ corresponding to a single beam splitter where $n$ 
photons are injected into one port with only the vacuum at the other port, generates $S U(2)$ coherent state. To generate a multipartite $S U(2)$ coherent states, we consider a $p$ uncoupled beam splitters acting on the Hilbert space $\mathcal{H}=\mathcal{H}_{1} \otimes \mathcal{H}_{2} \otimes \cdots \otimes \mathcal{H}_{2 p}$. This is a tensorial product of $2 p$ usual bosonic Fock space. The beam splitter $\mathcal{B}_{i, i+1}\left(\theta_{i}\right)$, with $i=1,3, \cdots, 2 p-1$, acts on the Fock space $\mathcal{H}_{i} \otimes \mathcal{H}_{i+1}$ as coupler of the modes $i$ and $i+1$. The action of this set of uncoupled beam splitters is described by the following transformation

$$
\mathcal{T}_{p, 1}=\mathcal{B}_{2 p-1,2 p}\left(\theta_{2 p-1}\right) \mathcal{B}_{2 p-3,2 p-2}\left(\theta_{2 p-3}\right) \cdots \mathcal{B}_{3,4}\left(\theta_{3}\right) \mathcal{B}_{1,2}\left(\theta_{1}\right)
$$

We assume that the input state of each beam splitter, characterized by the operation $\mathcal{B}_{i, i+1}\left(\theta_{i}\right)$, is $|n, 0\rangle \in \mathcal{H}_{i} \otimes \mathcal{H}_{i+1}$ where $n \in \mathbb{N}-\{0\}$ is an arbitrary photon number. Then, The input state of the entire system is given

$$
\mid \text { input }\rangle=|n, 0, n, 0, \cdots, n, 0\rangle,
$$

and one can see that the output state

$$
\left.\mathcal{T}_{p, 1} \mid \text { input }\right\rangle=\left|\alpha_{1}\right\rangle \otimes\left|\alpha_{3}\right\rangle \otimes \cdots \otimes\left|\alpha_{2 p-1}\right\rangle
$$

where $\left|\alpha_{i}\right\rangle(i=1,3, \cdots, 2 p-1)$ are the $S U(2)$ coherent states given by

$$
\left|\alpha_{i}\right\rangle=\left(1+\left|\alpha_{i}\right|^{2}\right)^{-\frac{n}{2}} \sum_{m=0}^{n} \frac{\sqrt{n !}}{\sqrt{(n-m) ! m !}} \alpha_{i}^{m}|n-m, m\rangle
$$

with the labeling parameter $\alpha_{i}=i r_{i} / t_{i}$ is expressed in terms of the reflection-transmission rate.

In a similar way, one can extend this scheme to generate states which are tensorial product of $S U(3)$ coherent states. This can be done as follows. We consider set of $p$ decoupled chain of beam splitters corresponding to $k=2$. Each $k=2$ chain of beam splitters is described by the unitary transformation $\mathcal{B}_{i+1, i+2}\left(\theta_{i+1}\right) \mathcal{B}_{i, i+1}\left(\theta_{i}\right)$ acting on the three particle Hilbert space $\mathcal{H}_{i} \otimes \mathcal{H}_{i+1} \otimes \mathcal{H}_{i+2}$ where $i$ takes the integer values $1,4,7, \cdots, 3 p-2$. It follows that the entire system is described by the following transformation

$$
\mathcal{T}_{p, 2}=\mathcal{B}_{3 p-1,3 p}\left(\theta_{3 p-1}\right) \mathcal{B}_{3 p-2,3 p-1}\left(\theta_{3 p-2}\right) \cdots \mathcal{B}_{5,6}\left(\theta_{5}\right) \mathcal{B}_{4,5}\left(\theta_{4}\right) \mathcal{B}_{2,3}\left(\theta_{2}\right) \mathcal{B}_{1,2}\left(\theta_{1}\right)
$$

The action of $\mathcal{T}_{p, 2}$ on the $3 p$-particles states of type $|n, 0,0, n, 0,0, \cdots, n, 0,0\rangle$ gives

$$
\mathcal{T}_{p, 2}|n, 0,0, n, 0,0, \cdots, n, 0,0\rangle=\left|\beta_{1}, \beta_{2}\right\rangle \otimes\left|\beta_{4}, \beta_{5}\right\rangle \otimes \cdots \otimes\left|\beta_{3 p-2}, \beta_{3 p-1}\right\rangle
$$

where $\left|\beta_{i}, \beta_{i+1}\right\rangle(i=1,4, \cdots, 3 p-2)$ are the $S U(3)$ coherent states defined in the previous subsection. Explicitly, they are given by

$$
\left|\beta_{i}, \beta_{i+1}\right\rangle=\left(1+\left|\beta_{i}\right|^{2}+\left|\beta_{i} \beta_{i+1}\right|^{2}\right)^{-\frac{n}{2}} \sum_{n_{i}=0}^{n} \sum_{n_{i+1}=0}^{n_{i}} \frac{\beta_{i}^{n_{i}} \beta_{i+1}^{n_{i+1}} \sqrt{n !}}{\sqrt{\left(n-n_{i}\right) !\left(n_{i}-n_{i+1}\right) ! n_{i+1} !}}\left|n-n_{i}, n_{i}-n_{i+1}, n_{i+1}\right\rangle
$$

with the labeling parameters defined by

$$
\beta_{i}=i t_{i+1} \frac{r_{i}}{t_{i}} \text { and } \quad \beta_{i+1}=i \frac{r_{i+1}}{t_{i+1}},
$$

are functions of transmission and reflection parameters.

This procedure to generate multipartite coherent states using a quantum network of beam splitters can be extended to obtain separable state involving the tensorial product of $S U(k+1)$ coherent states. 


\section{$3 \quad$ Entanglement of balanced superposition of $S U(2)$ multipartite coherent states}

The problem we raise in this section is the bipartite entanglement of the balanced superposition of two multi-particle coherent states. Before embarking on our study, it is worth making some remarks. First, it should be noted that for the most of tasks of quantum processing, one needs to generate such superpositions of multipartite $S U(2)$ coherent states. In the next subsection, we shall discuss their generation via Kerr nonlinearity. Second, to investigate the bipartite entanglement, we split the whole system in two subsystems. Also, we shall focus on the multi-particle $S U(2)$ coherent states given by (14) generated via the scheme described in the previous section. More precisely, we consider the superposed states of the form

$$
\left|\alpha ; \alpha^{\prime}\right\rangle \equiv \mathcal{N}_{p}\left[\left|\alpha_{1}\right\rangle \otimes\left|\alpha_{3}\right\rangle \otimes \cdots \otimes\left|\alpha_{2 p-1}\right\rangle+e^{i \theta}\left|\alpha_{1}^{\prime}\right\rangle \otimes\left|\alpha_{3}^{\prime}\right\rangle \otimes \cdots \otimes\left|\alpha_{2 p-1}^{\prime}\right\rangle\right]
$$

to study the bipartite entanglement. It is clear that this superposition is balanced or equally weighted.

The states $\left|\alpha_{1}\right\rangle \otimes\left|\alpha_{3}\right\rangle \otimes \cdots \otimes\left|\alpha_{2 p-1}\right\rangle$ and $\left|\alpha_{1}^{\prime}\right\rangle \otimes\left|\alpha_{3}^{\prime}\right\rangle \otimes \cdots \otimes\left|\alpha_{2 p-1}^{\prime}\right\rangle$ write as superpositions of vectors states of $2 p$ particles $\left|n_{1}, n_{2}, \cdots, n_{2 p-1}, n_{2 p}\right\rangle$. Remark that the analysis presented here can be generalized to the multipartite $S U(k+1)$ coherent states in a straightforward manner. The normalization constant in (19) is given by

$$
\mathcal{N}_{p}^{-2}=2\left(1+c_{1} c_{3} \cdots c_{2 p-1} \cos \theta\right)
$$

where the quantities $c_{i}$ for $i=1,3, \cdots, 2 p-1$ stand for the overlapping of $S U(2)$ coherent states. They are given by

$$
c_{i} \equiv c_{i}\left(\alpha_{i}, \alpha_{i}^{\prime}\right)=\left\langle\alpha_{i} \mid \alpha_{i}^{\prime}\right\rangle=\left(t_{i} t_{i}^{\prime}+r_{i} r_{i}^{\prime}\right)^{n}=\left[\cos \frac{\theta_{i}-\theta_{i}^{\prime}}{2}\right]^{n}
$$

in terms of the reflection-transmission coefficients.

We will employ the concurrence [33] as a measure of bipartite entanglement for the state $\left|\alpha ; \alpha^{\prime}\right\rangle$. We recall that for $\rho_{12}$ the density matrix for a pair of qubits 1 and 2 which may be pure or mixed, the concurrence is 33 .

$$
C_{12}=\max \left\{\lambda_{1}-\lambda_{2}-\lambda_{3}-\lambda_{4}, 0\right\}
$$

for $\lambda_{1} \geq \lambda_{2} \geq \lambda_{3} \geq \lambda_{4}$ the square roots of the eigenvalues of the "spin-flipped" density matrix

$$
\varrho_{12} \equiv \rho_{12}\left(\sigma_{y} \otimes \sigma_{y}\right) \rho_{12}^{\star}\left(\sigma_{y} \otimes \sigma_{y}\right)
$$

where the star stands for complex conjugation in the basis $\{|00\rangle,|01\rangle,|10\rangle,|11\rangle\}$ with the Pauli matrix is $\sigma_{y}=i|1\rangle\langle 0|-i| 0\rangle\langle 1|$. For the special case of pure state, one can show that the concurrence is $C_{12}=2 \sqrt{\operatorname{det} \rho_{1}}$, where $\rho_{1}$ is the reduced density of qubit 1 that is obtained by tracing out the second qubit. Nonzero concurrence occurs if and only if qubits 1 and 2 are entangled. Moreover, $C_{12}=0$ only for an unentangled state, and $C_{12}=1$ only for a maximally entangled state. This concurrence measure can be used to study the bipartite entanglement in multipartite coherent states for pure as well as mixed state as we shall explain below. 


\subsection{Generation of balanced superposition of multipartite $S U(2)$ coherent states}

As mentioned above, we shall discuss how to generate the balanced superposition of multipartite $S U(2)$ coherent states. We first focus on a single beam splitter $(k=1)$ with one port receives $n$ photons and the vacuum at the other. We assume that a Kerr medium is placed in the output of the beam splitter. The Kerr interaction is described by the interaction Hamiltonian

$$
H_{\mathrm{Kerr}}=\hbar \chi\left(a_{1}^{+} a_{1}^{-}\right)^{2}
$$

where $\chi$ is proportional to the third-order nonlinear susceptibility of the medium. Many authors employ an extended version where a linear term in the photon number $a_{1}^{+} a_{1}^{-}$is added. This linear term is irrelevant for our task. The unitary transformation associated with the Kerr interaction is

$$
U_{\text {Kerr }}(t)=\exp \left(-i t H_{\text {Kerr }} / \hbar\right)
$$

Clearly, the action of the beam splitter produces the $S U(2)$ coherent state $\left|\alpha_{1}\right\rangle$ (see equation (15)) where $\alpha_{1}$ is related to the transmission and reflection parameters of the device. In what follows we shall assume that the time $t$ for the light to cross the Kerr medium is such that $t=\pi / 2 \chi$. In this particular case, the action of the operator $U_{\mathrm{Kerr}}(t)$ on the output state of the beam splitter $\left|\alpha_{1}\right\rangle$ gives

$$
U_{\mathrm{Kerr}}(t)\left|\alpha_{1}\right\rangle=\frac{1}{\sqrt{2}}\left(e^{-i \frac{\pi}{4}}\left|\alpha_{1}\right\rangle+e^{+i \frac{\pi}{4}}\left|-\alpha_{1}\right\rangle\right)
$$

This can be generalized to generate some particular superposition of multi-component $S U(2)$ coherent states. For this end, we use the dynamical evolution of a tensorial product of $p S U(2)$ coherent states with respect the nonlinear Hamiltonian

$$
H=\hbar \chi\left(a_{1}^{+} a_{1}^{-}+a_{3}^{+} a_{3}^{-}+\cdots+a_{2 p}^{+} a_{2 p}^{-}\right)^{2} .
$$

This generalizes the single mode nonlinear Kerr hamiltonian. The eigenvalues of this Hamiltonian are

$$
H\left|n_{0}, n_{1}, n_{2}, n_{3}, \cdots, n_{2 p-1}, n_{2 p}\right\rangle=\hbar \chi\left(n_{1}+n_{3}+\cdots+n_{2 p}\right)^{2}\left|n_{0}, n_{1}, n_{2}, n_{3}, \cdots, n_{2 p-1}, n_{2 p}\right\rangle .
$$

Here also at the time $t=\frac{\pi}{2 \chi}$, the multipartite coherent state $\left|\alpha_{1}\right\rangle \otimes\left|\alpha_{3}\right\rangle \otimes \cdots \otimes\left|\alpha_{2 p-1}\right\rangle$ evolves into the state

$e^{-i t H / \hbar}\left|\alpha_{1}\right\rangle \otimes\left|\alpha_{3}\right\rangle \otimes \cdots \otimes\left|\alpha_{2 p-1}\right\rangle=\frac{1}{\sqrt{2}}\left(e^{-i \frac{\pi}{4}}\left|\alpha_{1}\right\rangle \otimes\left|\alpha_{3}\right\rangle \otimes \cdots \otimes\left|\alpha_{2 p-1}\right\rangle+e^{+i \frac{\pi}{4}}\left|-\alpha_{1}\right\rangle \otimes\left|-\alpha_{3}\right\rangle \otimes \cdots \otimes\left|-\alpha_{2 p-1}\right\rangle\right)$,

which is a superposition of two multipartite $S U(2)$ coherent states. It constitutes a special example of the balanced multipartite $S U(2)$ coherent states given by (19).

\subsection{Bipartite entanglement: Pure-state}

We now investigate the degree of bipartite entanglement in multipartite systems described by states of type $\left|\alpha ; \alpha^{\prime}\right\rangle$ defined by (19). Such states belong to a $2 p$ dimensional Hilbert space. As simple way to tackle this issue, one splits the entire system into two subsystems as follows

$$
\left.\left.\left.\left.\left|\alpha ; \alpha^{\prime}\right\rangle \equiv \mathcal{N}_{p}[\mid \alpha)_{q} \otimes \mid \alpha\right)_{p-q}+e^{i \theta} \mid \alpha^{\prime}\right)_{q} \otimes \mid \alpha^{\prime}\right)_{p-q}\right]
$$


where

$$
\left.\mid \alpha)_{q}=\left|\alpha_{1}\right\rangle \otimes\left|\alpha_{3}\right\rangle \otimes \cdots \otimes\left|\alpha_{2 q-1}\right\rangle \quad \mid \alpha\right)_{p-q}=\left|\alpha_{2 q+1}\right\rangle \otimes\left|\alpha_{2 q+3}\right\rangle \otimes \cdots \otimes\left|\alpha_{2 p-1}\right\rangle
$$

and

$$
\left.\left.\mid \alpha^{\prime}\right)_{q}=\left|\alpha_{1}^{\prime}\right\rangle \otimes\left|\alpha_{3}^{\prime}\right\rangle \otimes \cdots \otimes\left|\alpha_{2 q-1}^{\prime}\right\rangle \quad \mid \alpha^{\prime}\right)_{p-q}=\left|\alpha_{2 q+1}^{\prime}\right\rangle \otimes\left|\alpha_{2 q+3}^{\prime}\right\rangle \otimes \cdots \otimes\left|\alpha_{2 p-1}^{\prime}\right\rangle
$$

with $1 \leq q \leq p-1$. Thus, we consider the first $2 q$ systems as the subsystem 1 containing $q S U(2)$ coherent states and the other $2(p-q)$ systems as the subsystem 2 containing the remaining $p-q$ $S U(2)$ coherent states. Since the two subsystems in the bipartite state $\left|\alpha, \alpha^{\prime}\right\rangle$ are essentially two-state systems, we can characterize the entanglement of bipartite state by the bipartite concurrence that we denote by $\mathcal{C}_{q, p-q} \equiv \mathcal{C}_{(1,2 \cdots, 2 q)(2 q+1,2 q+2 \cdots, 2 p)}$. It follows that one can apply the method developed in [17, 19] to get the concurrence of a bipartite system involving nonorthogonal states. For that end, one has to write the multi-particle state $\left|\alpha, \alpha^{\prime}\right\rangle$ as a state of two logical qubits using the orthogonal basis $\left\{|\mathbf{0}\rangle_{q},|\mathbf{1}\rangle_{q}\right\}$ defined as

$$
\left.|\mathbf{0}\rangle_{q}=\mid \alpha\right)_{q}, \quad|\mathbf{1}\rangle_{q}=\frac{\left.\left.\mid \alpha^{\prime}\right)_{q}-c_{1} c_{3} \cdots c_{2 q-1} \mid \alpha\right)_{q}}{\sqrt{1-c_{1}^{2} c_{3}^{2} \cdots c_{2 q-1}^{2}}}
$$

for the first subsystem. Similarly, we introduce for the second subsystem the orthogonal basis $\left\{|\mathbf{0}\rangle_{p-q},|\mathbf{1}\rangle_{p-q}\right\}$ given by

$$
\left.|\mathbf{0}\rangle_{p-q}=\mid \alpha^{\prime}\right)_{p-q}, \quad|\mathbf{1}\rangle_{p-q}=\frac{\left.\mid \alpha)_{p-q}-c_{2 q+1} c_{2 q+3} \cdots c_{2 p-1} \mid \alpha^{\prime}\right)_{p-q}}{\sqrt{1-c_{2 q+1}^{2} c_{2 q+3}^{2} \cdots c_{2 p-1}^{2}}} .
$$

Reporting (25) and (26) in (24), we obtain the expression of the pure state density $\left|\alpha ; \alpha^{\prime}\right\rangle\left\langle\alpha ; \alpha^{\prime}\right|$ in the basis $\left\{|\mathbf{0}\rangle_{q} \otimes|\mathbf{0}\rangle_{p-q},|\mathbf{0}\rangle_{q} \otimes|\mathbf{1}\rangle_{p-q},|\mathbf{1}\rangle_{q} \otimes|\mathbf{0}\rangle_{p-q},|\mathbf{1}\rangle_{q} \otimes|\mathbf{1}\rangle_{p-q}\right\}$, from which one derives the reduced density matrix of the first component of the system. Then it is straightforward to check that the concurrence is given by

$$
\mathcal{C}_{q, p-q}=\frac{\sqrt{1-c_{1}^{2} c_{3}^{2} \cdots c_{2 q-1}^{2}} \sqrt{1-c_{2 q+1}^{2} c_{2 q+3}^{2} \cdots c_{2 p-1}^{2}}}{1+c_{1} c_{3} \cdots c_{2 p-1} \cos \theta} .
$$

Clearly, the separability condition of the state $\left|\alpha ; \alpha^{\prime}\right\rangle$ is provided by the condition $\mathcal{C}_{q, p-q}=0$. This implies

$$
c_{1} c_{3} \cdots c_{2 q-1}=1 \quad \text { or } \quad c_{2 q+1} c_{2 q+3} \cdots c_{2 p-1}=1 .
$$

Using the expression of the overlapping between two $S U(2)$ coherent states (20), it is simply verified that the first condition is satisfied when $c_{i}=1$ for each $i=1,3, \cdots, 2 q-1$. This means that $\alpha_{i}=\alpha_{i}^{\prime}$ or equivalently $\theta_{i}=\theta_{i}^{\prime}$ for all $i=1,3, \cdots, 2 q-1$. In this case the state $\left|\alpha ; \alpha^{\prime}\right\rangle$ is unentangled. Similarly, the second condition is satisfied when $\alpha_{i}=\alpha_{i}^{\prime}$ for $i=2 q+1,2 q+3, \cdots, 2 p-1$ and it is easy to see that the state $\left|\alpha ; \alpha^{\prime}\right\rangle$ is separable.

The state $\left|\alpha ; \alpha^{\prime}\right\rangle$ is entangled when $\mathcal{C}_{q, p-q} \neq 0$. From the expression of the concurrence (27), one can see that maximally entangled states are obtained for $\theta=\pi$. The minimal value of the concurrence is obtained for states with $\theta=0$ that we will call minimally entangled states.

At this level it is interesting to focus on some interesting particular cases. In this respect, We consider the state $\left|\alpha ; \alpha^{\prime}\right\rangle$ such that

$$
\theta_{1}-\theta_{1}^{\prime}=\theta_{3}-\theta_{3}^{\prime}=\cdots=\theta_{2 p-1}-\theta_{2 p-1}^{\prime}
$$


This condition, which can be realized by some appropriate choice of the variables labeling the $S U(2)$ coherent states, implies

$$
c_{1}=c_{3}=\cdots=c_{2 q-1}=c_{2 q+1}=c_{2 q+3}=\cdots=c_{2 p-1}=(\cos \varphi)^{n}
$$

where $\varphi=\left(\theta_{i}-\theta_{i}^{\prime}\right) / 2$ takes the same value for all $i=1,3, \cdots, 2 p-1$. It follows that the concurrence (27) takes the simple form

$$
\mathcal{C}_{q, p-q}=\frac{\sqrt{1-c^{2 n q}} \sqrt{1-c^{2 n(p-q)}}}{1+c^{n p} \cos \theta}
$$

where $c=\cos \varphi$.

For $p=2$, the only admissible value for $q$ is $q=1$. In this case, the equation (31) reads

$$
\mathcal{C}_{1,1}=\frac{1-c^{2 n}}{1+c^{2 n} \cos \theta}
$$

It is clear that for $\theta=\pi$, the state is maximally entangled. The concurrence $\mathcal{C}_{1,1}$ as function of the overlapping $c$ and $\theta$ when the input state contains a single photon $(n=1)$ is plotted in the figure 2 .

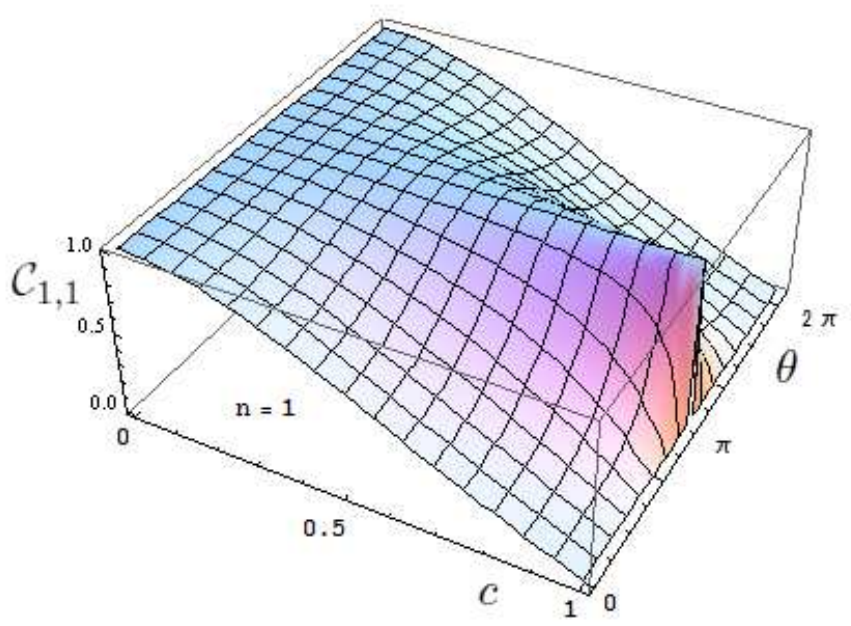

Fig. 2: The concurrence $\mathcal{C}_{1,1}$ as function of $c$ and $\theta$ for $n=1$.

For $\theta=0$, the states (24), obtained by the beam splitters satisfying the condition (29) or equivalently (30), are completely symmetric. In this case, we plot in figures 3 the concurrence $\mathcal{C}_{1,1}$ as function of $\varphi=\arccos c$ for $\theta=0$ and different input photon numbers $n$. It is remarkable that for $n$ increasing, the concurrence increases quickly to reach the maximal entanglement $\left(\mathcal{C}_{1,1}=1\right)$. 


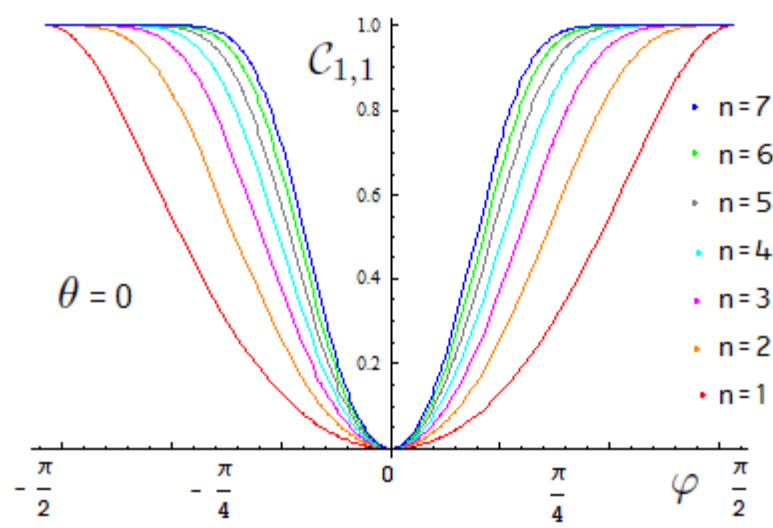

Fig. 3: The concurrence $\mathcal{C}_{1,1}$ as function of $\varphi=\arccos c$ for $\theta=0$ and different input photon numbers $n$.

Finally, we plotted also the $\mathcal{C}_{1,1}$ as function of $\varphi=\arccos c$ for $\theta=\frac{\pi}{2}$ and different input photon numbers $n$. This is given by the figure 4 . In this case, as it can be be seen from the figure, the concurrence increases with increasing number of input photons $n$.

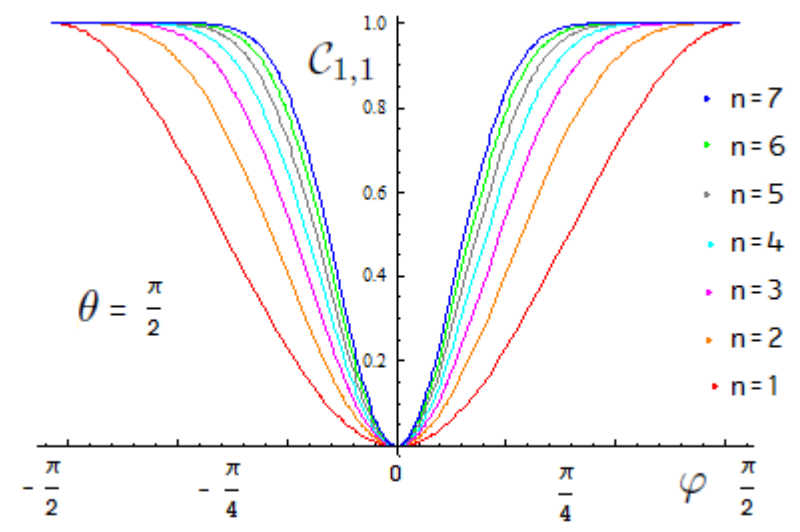

Fig. 4: The concurrence $\mathcal{C}_{1,1}$ as function of $\varphi=\arccos c$ for $\theta=\frac{\pi}{2}$ and different input photon numbers $n$.

As another illustration of the above analysis, we also consider the situation where $p=3$. In this case, one can divide the system such that $q=1$ or $q=2$. In this case, due to the symmetry property $\mathcal{C}_{q, p-q}=\mathcal{C}_{p-q, q}$, one has

$$
\mathcal{C}_{1,2}=\mathcal{C}_{2,1}=\frac{\sqrt{1-c^{2 n}} \sqrt{1-c^{4 n}}}{1+c^{3 n} \cos \theta}
$$

In figures 5, 6 and 7, we plot the concurrence $\mathcal{C}_{1,2}$ as function of $\varphi=\arccos c$ and $\theta$ for $n=1, n=5$ and $n=10$. 


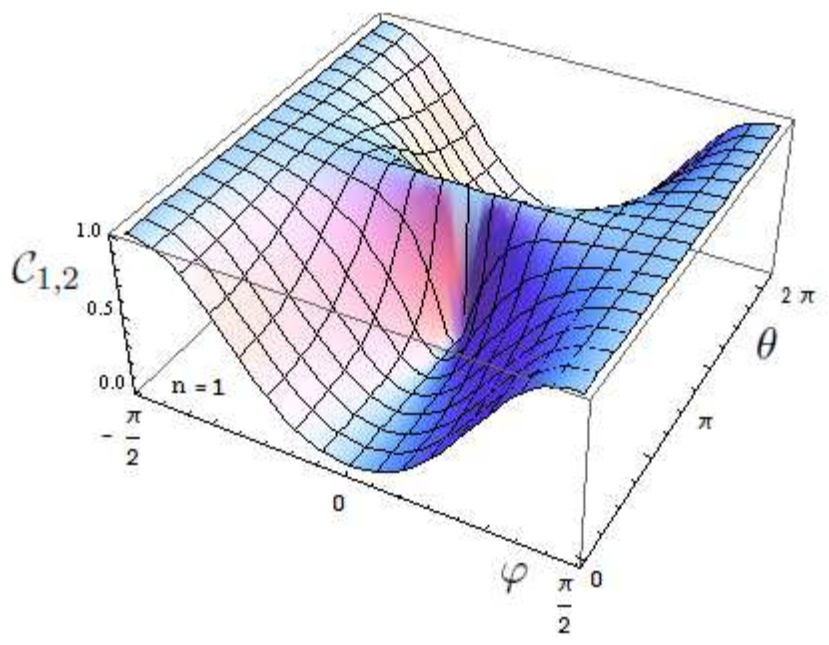

Fig. 5: The Concurrence $\mathcal{C}_{1,2}$ as function of $\varphi$ and $\theta$ for $n=1$.

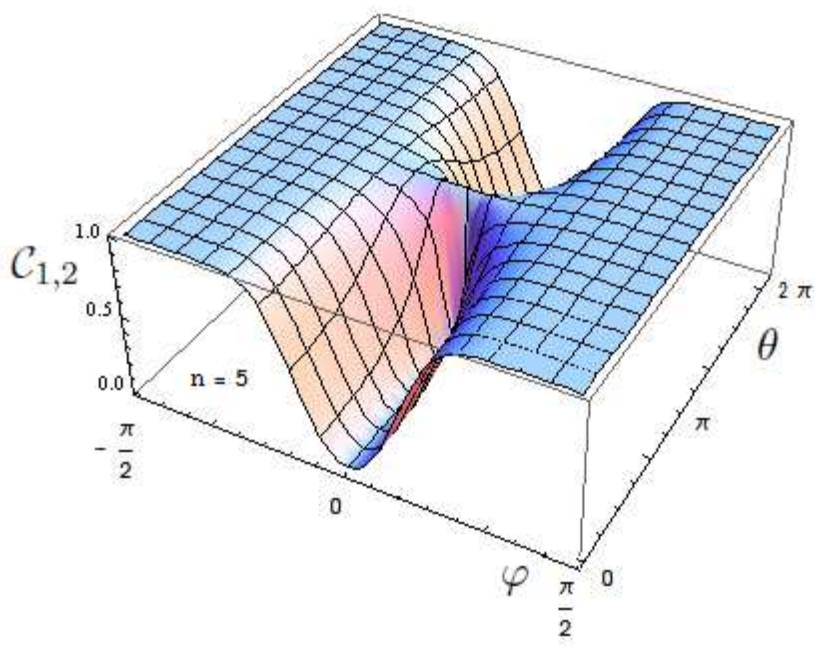

Fig. 6: The Concurrence $\mathcal{C}_{1,2}$ as function of $\varphi$ and $\theta$ for $n=5$.

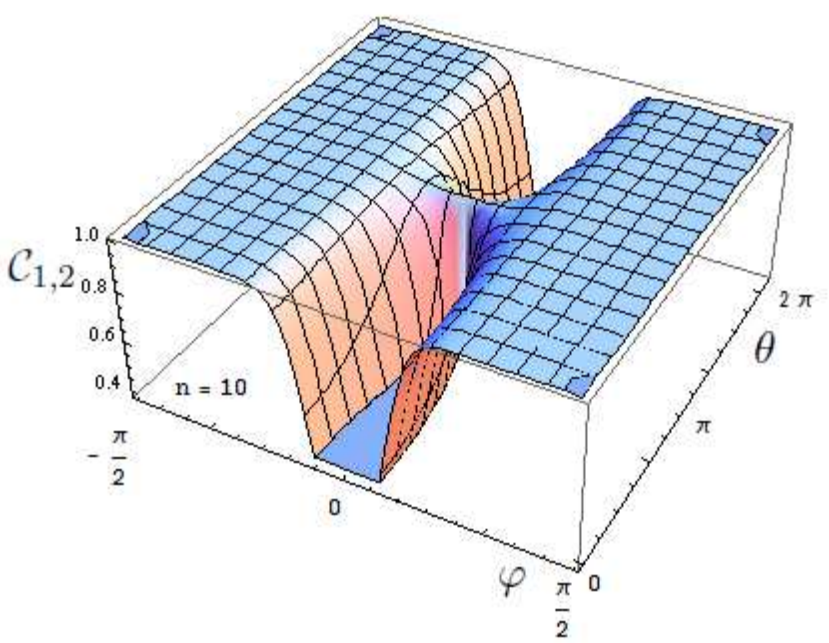


Fig. 7: The Concurrence $\mathcal{C}_{1,2}$ as function of $\varphi$ and $\theta$ for $n=10$.

\subsection{Bipartite entanglement: Mixed-state}

Another scheme to deal with the bipartite entanglement of the state $\left|\alpha ; \alpha^{\prime}\right\rangle$ can be achieved as follows. As $\left|\alpha ; \alpha^{\prime}\right\rangle$ is defined on a $2 p$ dimensional Hilbert space, a bipartite state can be defined by tracing out $(2 p-4)$ harmonic oscillator degrees of freedom

$$
\rho_{(1234)}=\operatorname{Tr}_{5, \cdots, 2 p}\left|\alpha ; \alpha^{\prime}\right\rangle\left\langle\alpha ; \alpha^{\prime}\right|
$$

It results that the reduced density matrix $\rho_{13} \equiv \rho_{(1234)}$ can be cast in the following form

$\rho_{13}=\mathcal{N}_{p}^{2}\left[\left|\alpha_{1}, \alpha_{3}\right\rangle\left\langle\alpha_{1}, \alpha_{3}\left|+c_{5} c_{7} \cdots c_{2 p-1} e^{-i \theta}\right| \alpha_{1}, \alpha_{3}\right\rangle\left\langle\alpha_{1}^{\prime}, \alpha_{3}^{\prime}\left|+c_{5} c_{7} \cdots c_{2 p-1} e^{+i \theta}\right| \alpha_{1}^{\prime}, \alpha_{3}^{\prime}\right\rangle\left\langle\alpha_{1}, \alpha_{3}|+| \alpha_{1}^{\prime}, \alpha_{3}^{\prime}\right\rangle\left\langle\alpha_{1}^{\prime}, \alpha_{3}^{\prime}\right|\right]$.

It only involves the $S U(2)$ coherent states labeled by the parameters $\alpha_{1}$ and $\alpha_{3}$ and it is indeed shared by two subsystems. The first (resp. second) is described by the bosonic degrees of freedom indexed by 1 and 2 (resp. 3 and 4 ).

Note that this is not the only way to introduce a bipartite density matrix and there are $p(p-1) / 2$ different density matrices $\rho_{k l}$. However, for our state $\left|\alpha ; \alpha^{\prime}\right\rangle$, all particles are equally entangled with each other and all the reduced density matrices $\rho_{k l}$ are identical. Therefore, it is sufficient to consider $\rho_{13}$ and to generalize from this case.

To simplify our purpose let us assume that the state $\left|\alpha ; \alpha^{\prime}\right\rangle$ is prepared such that $\alpha_{1}=\alpha_{3}^{\prime}$ and $\alpha_{3}=\alpha_{1}^{\prime}$. To obtain the concurrence (21), one has to diagonalize the density matrix (22). For this, we choose an orthogonal basis $\{|\mathbf{0}\rangle,|\mathbf{1}\rangle\}$ defined as

$$
|\mathbf{0}\rangle \equiv\left|\alpha_{1}\right\rangle, \quad|\mathbf{1}\rangle \equiv\left(\left|\alpha_{3}\right\rangle-\left\langle\alpha_{1} \mid \alpha_{3}\right\rangle|\mathbf{0}\rangle\right) / \sqrt{1-\left\langle\alpha_{1} \mid \alpha_{3}\right\rangle^{2}}
$$

It follows that substituting

$$
\left|\alpha_{1}\right\rangle=|\mathbf{0}\rangle \quad\left|\alpha_{3}\right\rangle=\sqrt{1-\left\langle\alpha_{1} \mid \alpha_{3}\right\rangle^{2}}|\mathbf{1}\rangle+\left\langle\alpha_{1} \mid \alpha_{3}\right\rangle|\mathbf{0}\rangle
$$

into Eq. (35), one has the density matrix $\rho_{13}$ in the basis $\{|\mathbf{0 0 0}\rangle,|\mathbf{0 1}\rangle,|\mathbf{1 0}\rangle,|\mathbf{1 1}\rangle\}$ and subsequently one obtains the "spin-flipped" density matrix $\varrho_{13}$ (defined as in (22) ). The square roots of eigenvalues of $\varrho_{13}$ are

$$
\lambda_{1}=\mathcal{N}_{p}^{2}\left(1-\left\langle\alpha_{1} \mid \alpha_{3}\right\rangle^{2}\right)\left(1+c_{5} c_{7} \cdots c_{2 p-1}\right), \lambda_{2}=\mathcal{N}_{p}^{2}\left(1-\left\langle\alpha_{1} \mid \alpha_{3}\right\rangle^{2}\right)\left(1-c_{5} c_{7} \cdots c_{2 p-1}\right), \lambda_{3}=\lambda_{4}=0
$$

and the concurrence is thus given by

$$
\mathcal{C}_{13}(p) \equiv \mathcal{C}_{(12)(34)}(p)=\frac{\left(1-\left\langle\alpha_{1} \mid \alpha_{3}\right\rangle^{2}\right) c_{5} c_{7} \cdots c_{2 p-1}}{1+c_{1} c_{3} \cdots c_{2 p-1} \cos \theta}
$$

Remark that, as we assumed $\alpha_{1}=\alpha_{3}^{\prime}$ and $\alpha_{3}=\alpha_{1}^{\prime}$, we have $c_{1}=c_{3}=\left\langle\alpha_{1} \mid \alpha_{3}\right\rangle$.

In the particular situation where $p=2$, the concurrence (39) reads as

$$
\mathcal{C}_{13}(p=2)=\frac{1-\left\langle\alpha_{1} \mid \alpha_{3}\right\rangle^{2}}{1+\left\langle\alpha_{1} \mid \alpha_{3}\right\rangle^{2} \cos \theta} .
$$


This quantity depends on the number $n$ of photons passing trough the beam splitters network (see equation (30) $)$, the difference phase orientations of the beam splitters 1 and 3 , i.e. $\theta_{1}-\theta_{3}$, and the parameter $\theta$. Indeed, the concurrence (40) can also be written in the form

$$
\mathcal{C}_{13}(p=2)=\frac{1-\left(\cos \theta_{13}\right)^{2 n}}{1+\left(\cos \theta_{13}\right)^{2 n} \cos \theta}
$$

where $\theta_{13}=\left(\theta_{1}-\theta_{3}\right) / 2$. It is clear that for $\theta_{13}=0$ or equivalently $\alpha_{1}=\alpha_{3}$, the concurrence vanishes. For fixed $\theta_{13}$, the the concurrence $\mathcal{C}_{13}(p=2)$ is maximal for $\theta=\pi$. It is also remarkable that there is a formal similarity between the expression $\mathcal{C}_{13}(p=2)$ and the concurrence $\mathcal{C}_{1,1}$ given by the equation (32) (modulo the substitution $\left.c=\cos \varphi \rightarrow \cos \theta_{13}\right)$. Hence, the behavior of the concurrence $\mathcal{C}_{13}(p=2)$ as function of $\cos \theta_{13}, \theta$ and the photon number $n$, initially injected in the network to generate the coherent states, is similar to one exhibited by the concurrence $\mathcal{C}_{1,1}$ (see the figures 2,3 and 4 ).

\section{Summary}

In view of the first part of the paper we can state that any $S U(k+1)$ coherent state in the Perelomov sense can be generated by using a network composed by $k$ beam splitters. The continuous parameters labeling such states are expressed in term of the reflection-transmission parameters of the considered beam splitters. Based on this result, we have discussed the bipartite entanglement of a balanced superposition of multipartite coherent states. We particularly investigated the bipartite entanglement of multi-component $S U(2)$ coherent states. We discussed the pure as well as the mixed states cases. We gave the evolution of the concurrence as function of the number of photons initially injected in the network, the transmission parameters of the beam splitters. This is corroborated by numerical analysis in some particular situations. This shows clearly that the generation of multipartite entangled coherent can simply be achieved using a network of beam splitters.

\section{Acknowledgments:}

MD would like to express his thanks to Max Planck Institute for Physics of Complex Systems (DresdenGermany) where this work was done.

\section{References}

[1] C.H. Bennett, G. Brassard, C, Crépeau, R. Jozsa, A. Peres and W.K. Wootters, Phys. Rev. Lett. 70 (1993) 1895.

[2] C.H. Bennett and S.J. Wiesner, Phys. Rev. Lett. 69 (1992) 2881.

[3] A.K. Ekert, Phys. Rev. Lett. 67 (1991) 661.

[4] C.A. Fuchs, Phys. Rev. Lett. 79 (1997) 1162.

[5] R. Rausschendorf and H. Briegel, quant-ph/0010033. 
[6] D. Gottesman and I. Chuang, Nature 402 (1999) (6760) 390.

[7] P. Rungta, V. Buzek, C.M. Caves, M. Hillery, and G.J. Milburn, Phys. Rev. A 64 (2001) 042315.

[8] C.H. Bennett, D.P. DiVincenzo, J. Smolin, and W.K. Wootters, Phys. Rev. A 54 (1996) 3824.

[9] W.K. Wootters, Phys. Rev. Lett. 80 (1998) 2245.

[10] V. Coffman, J. Kundu, and W.K. Wootters, Phys. Rev. A 61 (2000) 052306.

[11] P.G. Kwiat, S. Barraza-Lopez, A. Stefanov and N. Gisin, Nature 409 (2001) 1014.

[12] S.M. Tan, D.F. Walls and M.J. Collett, Phys. Rev. Lett. 66 (1991) 252.

[13] B.C. Sanders, Phys. Rev. A 45 (1992) 6811.

[14] B.C. Sanders, K.S. Lee and M.S. Kim, Phys. Rev. A 52 (1995) 735.

[15] M.G.A. Paris, Phys. Rev. A 59 (1999) 1615.

[16] M.S. Kim, W. Son, V.Buzek and P.L. Knight, Phys. Rev. A 65 (2002) 032323.

[17] A. Mann, B. C. Sanders, and W. J. Munro, Phys. Rev. A 51, 989 (1995).

[18] X. Wang, B.C. Sanders and S.H. Pan, J. Phys. A: Math. Gen. 33 (2000) 7451.

[19] H. Fu, X. Wang and A. I. Solomon, Phys. Lett. A, 291 (2001) 73.

[20] S.J. van Enk, O. Hirota, Phys. Rev. A 64 (2001) 022313.

[21] H. Jeong, M.S. Kim, J. Lee, Phys. Rev. A 64 (2001) 052308.

[22] H. Jeong, M.S. Kim, Phys. Rev. A 65 (2002) 042305.

[23] M. Yang, Z.-L. Cao, Physica A 366 (2006) 243.

[24] N. Sangouard, C. Simon, N. Gisin, J. Laurat, R. Tualle-Brouri and P. Grangier, J. Opt. Soc. Am. B 27 (2010) A137.

[25] D. Markham and V. Vedral, Phys. Rev. A 67 (2003) 042113.

[26] C.C. Gerry and A. Benmoussa, Phys. Rev. A 71 (2005) 062319.

[27] J.R. Klauder and B.S. Skagerstam, Coherent states-Applications in Physics and Mathematical Physics (World Scientific,Singapore,1985).

[28] A. Perelomov, Generalized Coherent States and their Applications,Texts and Monographs in Physics, (Spinger-Verlag,1986).

[29] C.C. Gerry, Phys. Rev. A 59 (1999) 4095.

[30] A. Luis, Phys. Rev. A 64 (2001) 054102. 
[31] X. Wang and B.C. Sanders, Phys. Rev. A 65 (2002) 012303.

[32] X. Wang, J. Phys. A: Math. Gen. 35 (2002) 165.

[33] S. Hill and W. K. Wootters, Phys. Rev. Lett. 78 (1997) 5022; W. K. Wootters, Phys. Rev. Lett. 80 (1998) 2245.

[34] P. van Loock and S.L. Braunstein, Phys. Rev. Lett. 84 (2000) 3482.

[35] M. Daoud, Phys. Lett. A 329 (2004) 318.

[36] S. Brattke, B.T.H. Varcoe and H. Walther, Phys. Rev. Lett. 86 (2001) 3534.

[37] M. Hofheinz, E.M. Weig, M. Ansmann, R.C. Bialczak, E. Lucero, M. Neeley, A.D. O’Connell, H. Wang, J.M. Martinis and A.N. Cleland, Nature 454 (2008) 310. 